\title{
ARQUEOMALACOLOGÍA EN EL SITIO ARQUEOLÓGICO POZO DE LA CHOLA (REGIÓN DEL PIEDEMONTE DE JUJUY, ARGENTINA). ESTUDIOS TAFONÓMICOS, INFERENCIAS AMBIENTALES Y PROCESOS ANTRÓPICOS
}

\author{
ARCHAEOMALACOLOGY AT THE ARCHAEOLOGICAL SITE POZO DE LA \\ CHOLA (FOOTHILL REGION OF JUJUY, ARGENTINA). TAFONOMIC STUDIES, \\ ENVIRONMENTAL INFERENCES AND ANTHROPIC PROCESSES.
}

\author{
Gabriela Ortiz ${ }^{1}$, Alvaro Alavar 2, Verónica Nahir Rojas³, Nelly Vargas Rodríguez ${ }^{4}$ y \\ Franco Quispe ${ }^{5}$ \\ ${ }^{1}$ CISOR-CONICET-Universidad Nacional de Jujuy, C/San Martín 1208. San \\ Salvador de Jujuy, Argentina. yolatordo@hotmail.com; \\ ${ }^{2}$ CISOR-CONICET-Universidad Nacional de Jujuy, C/San Martín 1208. San \\ Salvador de Jujuy, Argentina. alvarojosealavar@gmail.com \\ ${ }^{3}$ CREA-FHyCS. Universidad Nacional de Jujuy, C/San Martín 1208. San Salvador \\ de Jujuy, Argentina. veronica.nahir.jujuy@hotmail.com; \\ ${ }^{4}$ LALIMECO- FCA. Universidad Nacional de Jujuy, C/ Alberdi 47. San Salvador \\ de Jujuy, Argentina. nelly_vargas@yahoo.com. \\ ${ }^{5}$ CISOR-CONICET-Universidad Nacional de Jujuy, C/San Martín 1208. San \\ Salvador de Jujuy, Argentina. francoalejandroxp@gmail.com.
}

Presentado: 06/03/2019 - Aceptado: 04/05/2019

\section{Resumen}

Restos de moluscos gasterópodos son frecuentes en los niveles fértiles de los sitios arqueológicos de la región del piedemonte de Jujuy en el noroeste de Argentina, asignados al período Formativo temprano (500 AC-600 DC), sin embargo, análisis de esta clase de fauna no habían sido abordados hasta el año 2014, en donde se realiza una primera interpretación acerca de su presencia en los contextos excavados y sus implicancias económicas. En esta oportunidad, con el objeto de discernir cuestiones relacionadas a los diversos procesos involucrados con la existencia de moluscos en los niveles arqueológicos (tafonómicos y antrópicos), se presentan los resultados obtenidos en el sitio Pozo de la Chola (región del río San Francisco, Jujuy, Argentina), a escala espacial intrasitio. Se evaluaron las diferentes clases de distribuciones tanto verticales como horizontales en sectores discretos de excavación, así como en los 105 pozos de sondeos realizados para establecer la dimensión del sitio arqueológico. Se evaluaron distintos indicadores tales como; etología de los moluscos, perfiles biométricos, asociación con otras clases de arqueofaunas y de materiales arqueológicos, presencia/ausencia de diferentes agentes tafonómicos y antrópicos, distribuciones espaciales. Los resultados indican una notable prevalencia de los gasterópodos de la familia 
Ampullariidae que tienden a concentrarse en algunos sectores específicos del sitio, particularmente aquellos vinculados con áreas de procesamiento.

Palabras Claves: Región Pedemontana, Tradición San Francisco, Arqueomalacología

\begin{abstract}
Remains of gastropod mollusks are frequent at fertile levels of the archaeological sites in the foothill region of Jujuy in northwestern Argentina, assigned to the early Formative Period (500 BC-600 AD). However, analysis of this type of fauna had not been approached until 2014, where a first interpretation was made about its presence in the excavated contexts and its economic implications. In this opportunity, in order to discern related issues to the various processes involved with the existence of mollusks at the archaeological levels (taphonomic and anthropic), the study presents the obtained results at the site Pozo de la Chola (San Francisco River region, Jujuy, Argentina), at intrasite spatial scale. Different kinds of vertical and horizontal distributions in discrete excavation sectors were evaluated, as well as in the 105 boreholes, in order to establish the dimension of the archaeological site. Various indicators were evaluated, such as ethology of mollusks, biometric profiles, association with other classes of archaeofauna and archaeological materials, presence/absence of different taphonomic and anthropic agents and spatial distributions. The results indicate a notable prevalence of gastropods of the family Ampullariidae, which tend to concentrate in some specific sectors of the site, particularly those linked to processing areas.
\end{abstract}

Key Words: Foothill region, San Francisco Tradition, Archaeomalacology

\title{
Antecedentes
}

La región del valle del río San Francisco, se encuentra emplazada al oriente de la provincia de Jujuy, entre las estribaciones montañosas de las Sierras Subandinas del noroeste de Argentina (NOA). Se trata de un amplio valle inter montano con una importante red fluvial, y cursos de agua de régimen permanente cuyos ríos principales se convierten en afluentes del río Bermejo, en la macro-cuenca del Paraná-Plata. Los dos ríos más importantes son el Grande y el Lavayén; ambos confluyen en la latitud $24^{\circ} 1131^{\prime \prime}$, y forman el denominado río San Francisco, que da su nombre al valle (Figura 1). El clima imperante es subtropical con estación seca en invierno. Las temperaturas promedio son, arriba de $\operatorname{los} 28^{\circ} \mathrm{C}$, llegando incluso en verano a alcanzar temperaturas por encima de $\operatorname{los} 36^{\circ} \mathrm{C}$. Fitogeográficamente pertenece a dos distritos; el bosque chaqueño, que domina en el fondo de valle y el de Yunga o selva de montaña presente a medida que se asciende por las laderas de los cerros. Esto le confiere particularidades en lo que respecta a la existencia de animales y vegetales de ambos distritos configurando un ambiente con una muy alta biodiversidad. En relación a la malacofauna moderna gasterópos fluviales y terrestres son comunes, así como bivalvos fluviales. Las especies que han sido reconocidas hasta el momento y consideradas endémicas son: Megalobulimus oblongus aff. lorentzianus (Doering 1877); 2) Bulimulus aff. 
apodemetes (d'Orbygni 1835); 3) Epiphragmophora aff. trigrammephora (d'Orbygni 1835); 4) Pomacea aff. canaliculata (Lamarck 1828); y 5) Anodontites aff. trapesialis (Lamarck 1819).

En lo que respecta a la ocupación humana, los datos fehacientes conocidos hasta el momento indican la presencia de una población desde comienzos de la era cristiana, que habría ocupado no solo este extenso valle, sino también, otras regiones aledañas incluso, afuera de los límites actuales de la provincia de Jujuy. Dividido en tres sectores, la porción media y alta del valle estuvo ocupada desde aproximadamente la primera mitad del primer milenio antes de Cristo, con una permanencia ininterrumpida por parte de las mismas poblaciones durante un lapso de más de 1.000 años (Ortiz 2007; Ortiz y Nieva 2014). Fueron denominadas en la jerga arqueológica como pertenecientes a la "tradición San Francisco", sobre la base de su materialidad cerámica especialmente. Los sitios muestran una orientación fluvial en el patrón de asentamiento y algunos parecen haber sido habitados durante varios siglos a juzgar por los fechados radiocarbónicos (Ortiz y Killian Galván 2016; Ortiz 2015).

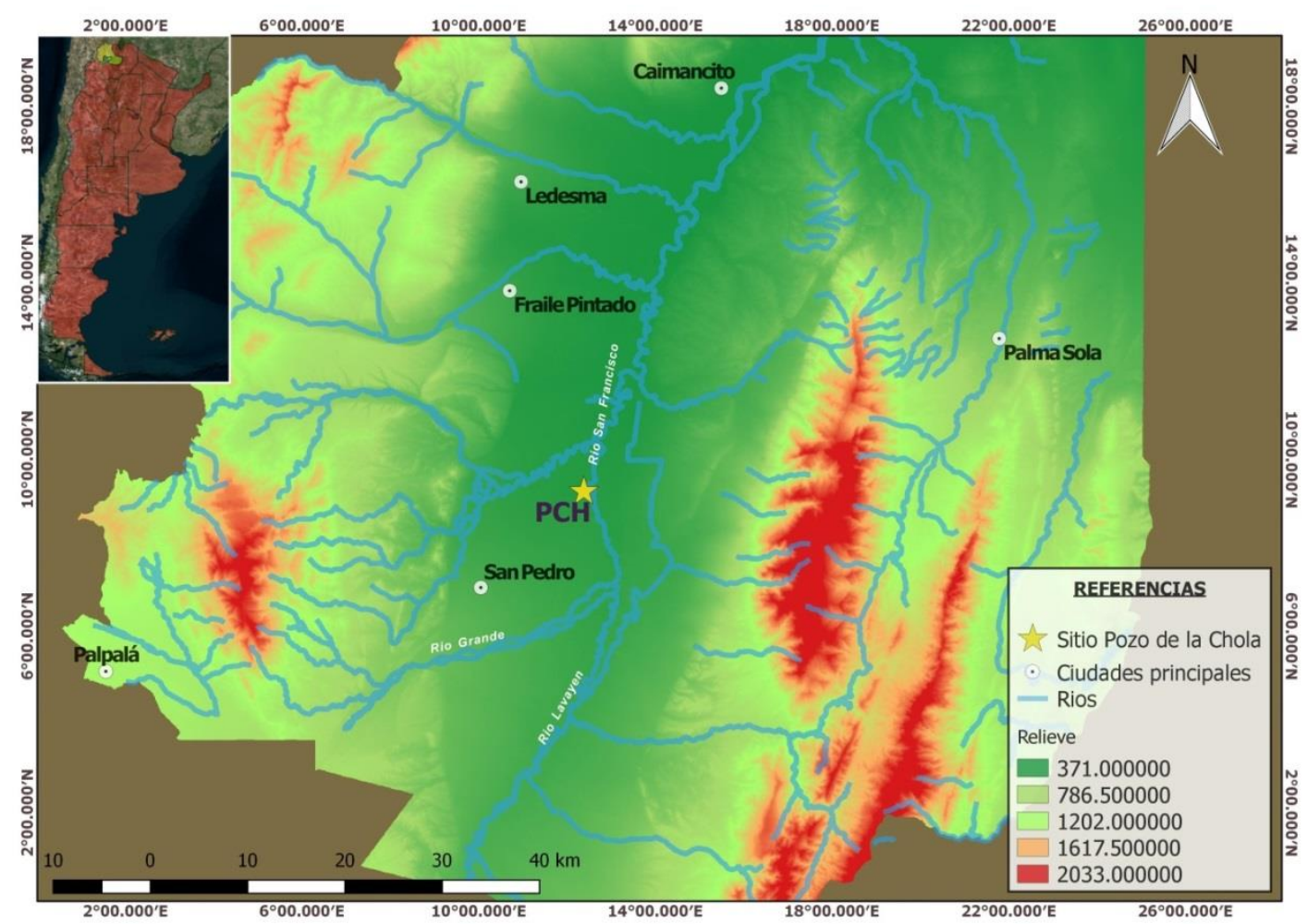

Figura 1. Mapa de la región del río San Francisco y la localización del sitio Pozo de la Chola.

El sitio Pozo de la Chola, se encuentra emplazado a la orilla del río San Francisco sobre una terraza fluvial de $2 \mathrm{~m}$ de potencia promedio (S24 $06^{\prime} 56^{\prime \prime}$ y $\mathrm{O} 64^{\circ} 42^{\prime} 59^{\prime \prime}$ ), en un suelo clasificado como Idisol de una sub-cuenca bien drenada (Figura 1), con una vegetación dominante de tipo bosque chaqueño. Los materiales arqueológicos en el sitio se presentan parcialmente expuestos en un extenso perfil de una de las terrazas del río con una extensión 
observable de $300 \mathrm{~m}$. Considerando que la visibilidad es nula y sumado a la ausencia de arquitectura en superficie, una exploración previa basada en una metodología prospectiva sistemática, permitió identificar algunos sectores con mayor concentración de artefactos y ecofactos. Excavaciones realizadas en el sitio entre los años 2010 a 2013 permitieron detectar la existencia de diferentes especies de fauna malacológica en los diversos estratos fértiles y en los pisos de ocupación, las que pudieron ser asignadas a diferentes categorías taxonómicas (Ortiz y Vargas Rodríguez 2015; Ortiz y Killian Galván 2016; Ortiz et al. 2015). Los primeros análisis al respecto permitieron plantear la hipótesis de que la presencia de algunas de las especies de esta clase de arqueofauna tendría su origen en una selección antrópica orientada al consumo, y muy escasos restos fueron interpretados como artefactos o como intrusivos. Con el fin de testear estas primeras interpretaciones, nuevos datos fueron incorporados, incluyendo aquellos de otros contextos excavados en el sitio, así como los obtenidos de distintos pozos de sondeos que fueron realizados para estimar los límites del área de ocupación.

\section{Materiales y métodos}

Con el objeto de constatar la distribución de materiales arqueológicos y estimar la dimensión total del asentamiento, se planteó un diseño de sondeos sistemáticos en una grilla ortogonal, que fue trazada sobre la base de la información recuperada a partir de algunos sondeos con barreno agronómico realizados en 3 transectas prospectivas previas. La grilla cubrió una extensión de 300 m con orientación N-S y 150 m E-O (45.000 m²), sobre la base de las observaciones realizadas en el perfil expuesto de la terraza del río. En los puntos de intersección, cada $20 \mathrm{~m}$, se realizaron sondeos de pala de $50 \times 50 \mathrm{~cm}$ por niveles artificiales de 10 $\mathrm{cm}$ hasta alcanzar una profundidad de 0,80 m ( $\mathrm{N}=105)$ (Figura 2). Los hallazgos fueron registrados por nivel y se obtuvo una muestra para ser tamizada mediante el método de zaranda húmeda en los niveles excavados (se tamizaron un total de 274 litros de sedimento). También se utilizó zaranda seca, a los efectos de recuperar la mayor cantidad de vestigios (incluyendo fauna y restos vegetales). La información fue procesada con programas de información geográfica (SIG) con el objeto de evaluar distintos tipos de distribuciones espaciales de los materiales recuperados (cerámica $(\mathrm{N}=1745)$, lítico $(\mathrm{N}=304)$, carbones $(\mathrm{N}=119)$, entre otros). A partir de este trabajo se pudo estimar el tamaño aproximado del área ocupada, revelando una mayor envergadura que la considerada hasta el momento, con una extensión de aproximadamente 2,5 ha. para el sector conservado del sitio, ya que una importante crecida estacional del río, habría destruido parte del mismo. También permitió observar las diferentes distribuciones y concentraciones de restos arqueológicos evaluando posibles sectores de actividades intrasitio (Figura 3).

Sobre la base de esta información, se planteó la excavación de nuevos sectores. Hasta el momento, se han excavado en área en el sitio 5 lugares diferentes, los que se encuentran emplazados en distintas Unidades de Procedencia (UP) (Figura 4). En cada uno de ellos se 
han recuperado diferentes especies de moluscos asociados a los pisos de ocupación, pero con disímiles distribuciones y frecuencias. Los datos analizados en esta oportunidad (específicamente de la excavación en las UP B y C), y de los sondeos exploratorios de control, fueron comparados con aquellos reportados para la UP A en un trabajo previo (Ortiz y Vargas Rodríguez 2015).
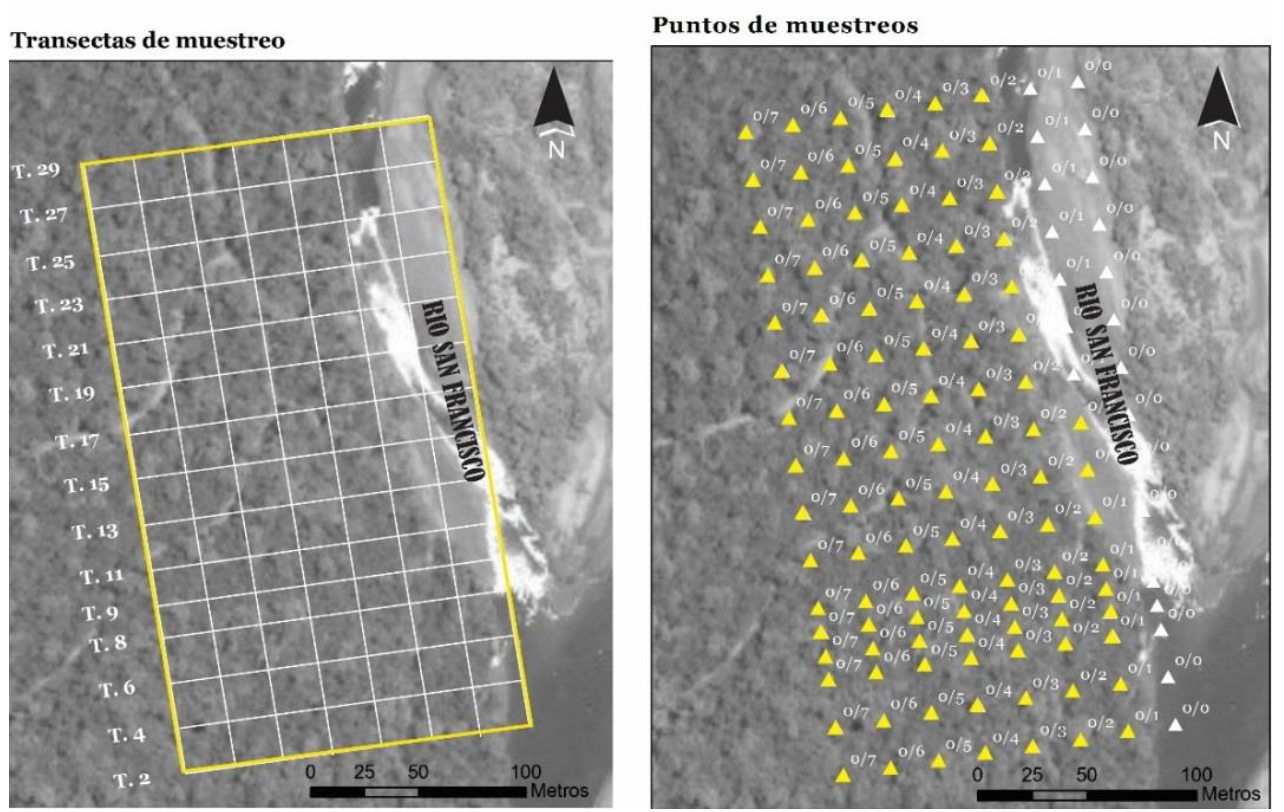

Figura 2. Localización de la grilla de sondeos con indicación de los diferentes puntos realizados de muestreo en color amarillo.

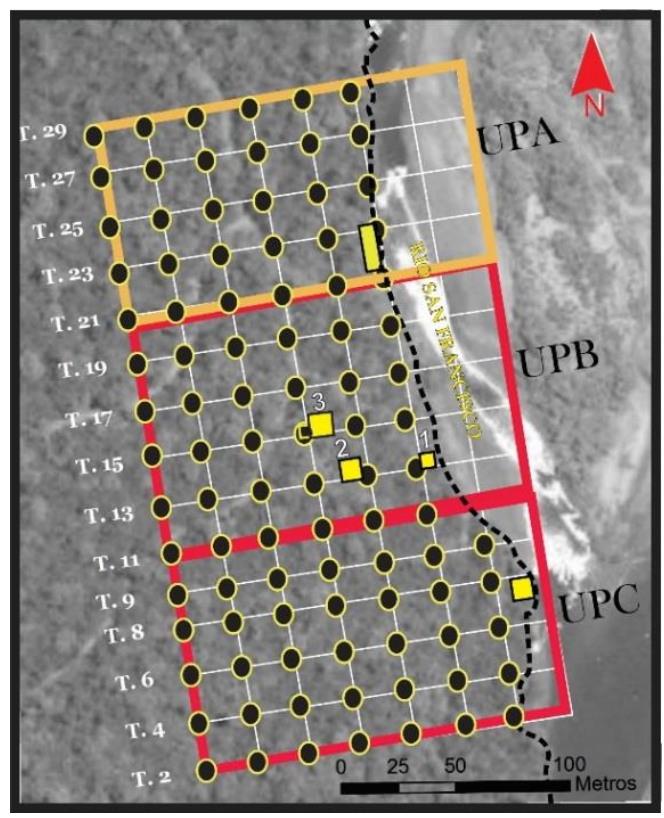

Figura 3. Mapas de calor que indican los lugares con mayores concentraciones de artefactos y ecofactos en el sitio arqueológico. Cerámica ( $\mathrm{N}=1745)$, Lítico $(\mathrm{N}=304)$, Carbones $(\mathrm{N}=119)$. 


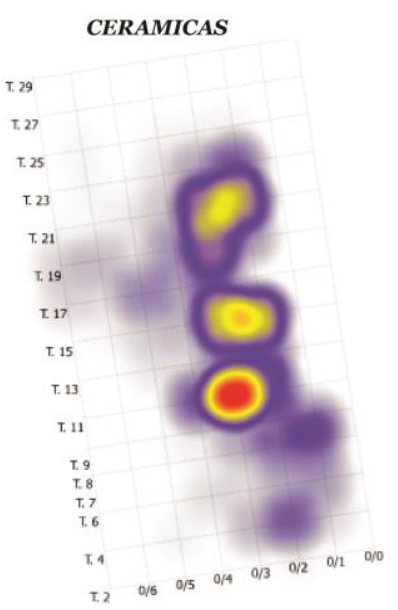

Sin hallazgos

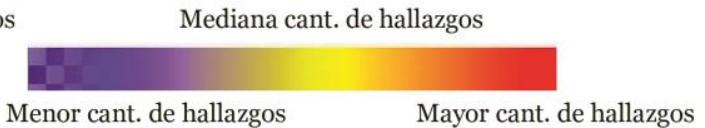

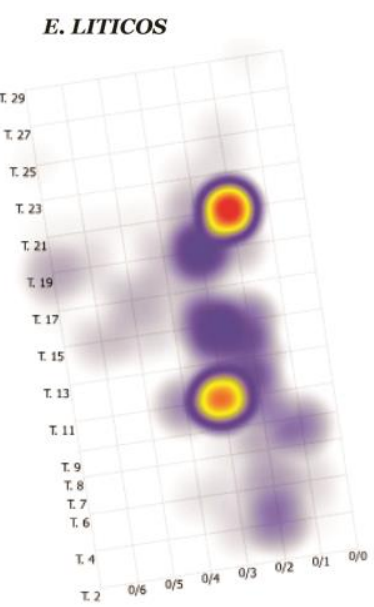

Mayor cant. de hallazgos

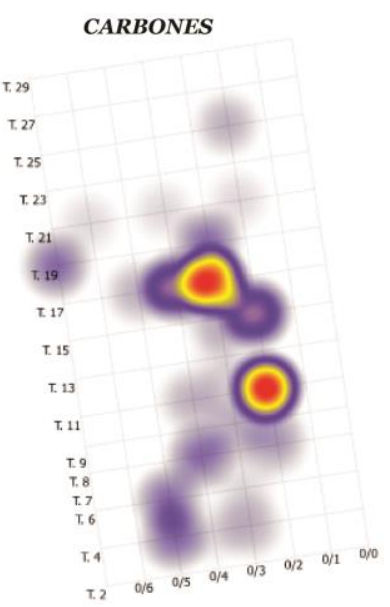

$150 \mathrm{~m}$

Figura 4. Se indican en color amarillo los diferentes sectores excavados en área y las Unidades de procedencia, sobre la grilla de muestreo en el sitio arqueológico.

Para la determinación taxonómica de la malacofauna se tuvo en cuenta la morfología exclusivamente, tomando en consideración los principales caracteres taxonómicos: estructura de la charnela, ornamentación de las valvas, estructura de la espira, abertura, características de la capa nacarada. Además se tomaron las medidas de la longitud total (desde el ápex hasta la abertura) y el ancho máximo, con un calibre vernier. Para la identificación se utilizaron las claves y referencias regionales, nacionales e internacionales (Gordillo 2010; Miquel y Aguirre 2011; Ramírez et al. 2012; Vázquez Silva et al. 2011). Se realizaron algunas comparaciones con el material actual de referencia (Bulimulidae, Ampullariidae) depositado en la colección malacológica del Laboratorio de Limnología y Ecología Acuática, Cátedra de Ecología, Facultad de Ciencias Agrarias y del laboratorio del CREA, Facultad de Humanidades y Ciencias Sociales, ambos de la Universidad Nacional de Jujuy. Luego de la determinación taxonómica se realizó el recuento de la abundancia por especie para determinar algunos parámetros poblacionales de la muestra.

Se llevó a cabo la estimación del NISP (número de especímenes identificados por especie) y el NMI (número mínimo de individuos). Para este último cálculo se aplicó la fórmula propuesta por Bejega García (2009), que permite estimar con mayor precisión la cantidad de individuos por especie sobre la base de la cuantificación de ejemplares completos y fragmentados, y de acuerdo a las diferentes categorías de fragmentación para 
gasterópodos. La fórmula utilizada fue ICOM+IFRA+FEST+FUMB; siendo ICOM (individuos completos); IFRA (individuos fragmentados); FEST (fragmento apical) y FUMB (fragmento umbilical). Para evaluar la posible existencia de selección por tamaño en los gasterópodos se midieron todos los ejemplares teniendo en cuenta el largo total y alto de la espira, y se realizó el cálculo del promedio. También se consideraron valores de abundancia relativa para cada especie.

Sobre la base de la información biométrica de las dimensiones que alcanzan las distintas especies, se consideraron 4 intervalos de tamaños, que abarcan desde 0,5 a 15 $\mathrm{mm}$; 16 a $25 \mathrm{~mm} ; 26$ a $40 \mathrm{~mm} ; 41$ a $60 \mathrm{~mm}$. A partir de las mediciones obtenidas se realizaron gráficos de dispersión, a los efectos de evaluar los tamaños más representados. Se consideraron las abundancias relativas por nivel excavado a escala espacial del sitio como de cada Unidad de Procedencia. Esto con el objeto de estimar tanto los procesos de formación de sitio como las implicancias cronológicas de la presencia de cada una de las especies de malacofauna en los diferentes niveles de agregación. Además de la identificación de las especies se contemplaron diversos indicadores con el fin de analizar factores tafonómicos en la conformación y distribución de la muestra. Entre ellos, características etológicas de cada especie (hábitos, distribución, etc.); así como las causas naturales que inciden en la representación, preservación y distribución; por ej. agentes bioerosionadores, disolución o conversión química, agentes de transporte y depredadores.

Posteriormente se establecieron Grupos tafonómicos de acuerdo a los criterios establecidos por diferentes autores (Gautier 1987; Moreno Nuño 1994; Gutiérrez Zugasti 2008-2009; Villamarzo 2010), los que ya fueron utilizados en un trabajo anterior (Ortiz y Vargas Rodríguez 2015). Estos contemplan los siguientes grupos no excluyentes entre sí: a) Restos alimentarios (incluye los restos potencialmente consumibles); b) Restos modificados (incluye las conchas modificadas por el hombre para la fabricación de utensilios, ornamentos,...o las que presentan modificaciones debidas a agentes naturales que por su morfología o características generales permitan su posterior uso por parte del ser humano; c) Restos de funcionalidad antrópica desconocida (restos recogidos por el hombre cuando el animal ya ha muerto y que no presentan ningún tipo de modificación que permita inferir claramente su función); y d) Restos intrusivos (restos que no han sido acumulados intencionalmente por el ser humano). Estos últimos se diferencian a su vez en dos clases; Intrusivos penecontemporáneos e intrusivos posteriores. Los primeros se depositaron al mismo tiempo que el resto del material que contiene el depósito arqueológico, ya sea por encontrarse en el terreno o por ser traídos junto a algún resto arqueológico, vegetal, animal o mineral. Los segundos fueron depositados con posterioridad a la formación del depósito (Bejega García 2009:69; Gutiérrez Zugasti 2008-2009). 
La información sobre situación de hallazgo (localización horizontal y distribución vertical) para las diferentes especies identificadas, fue ingresada en planillas de cálculo Excel y convertida a planillas shape, para ser procesada con el programa Qgis, y representada mediante mapas de calor, a los efectos de evaluar espacialmente las distintas distribuciones y frecuencias de esta clase de fauna, así como comparar su distribución con la de otras clases de arqueofauna.

\section{Las áreas excavadas}

Sector UPB

En esta Unidad de Procedencia se han excavado hasta el momento 3 sectores. El primero de ellos abarca una superficie de $6 \mathrm{~m}^{2}$, y constituye un área exclusiva de inhumación con ausencia de cualquier evidencia que indique que se hubieran desarrollado otra clase de actividades (Ortiz et al. 2018). Los restos cerámicos corresponden a distintos fragmentos de tamaño pequeño distribuidos aleatoriamente a diferentes profundidades como resultado del cavado de las fosas de inhumación y su posterior relleno. Cada individuo tenía colocada una vasija cerámica a la manera de acompañamiento mortuorio. Los restos arqueofaunísticos son escasos y se registró una cantidad muy grande de placas de dasipódidos en el nivel superior.

El segundo, denominado UP B2, abarcó una extensión total de $16 \mathrm{~m}^{2}$. Se excavó hasta alcanzar nivel estéril a los 0,70 $\mathrm{m}$ de profundidad. La abundante cantidad de diferentes clases de artefactos y desechos, indica un área donde se habrían llevado a cabo numerosas actividades que posiblemente incluyan la preparación de alimentos y descarte de materiales cerámicos. Se detectó un piso a los 0,35 - 0,40 m de profundidad. Se recuperaron una gran cantidad de restos óseos de fauna con una estimación de NR (número total de restos) de 3.286, incluido los moluscos. Entre otros hallazgos de interés podemos destacar, dos artefactos de cobre; una punta lítica de obsidiana, macrorestos botánicos carbonizados de especies silvestres y domésticas (Alavar 2017), 6.045 fragmentos de diferentes piezas cerámicas, artefactos líticos de cuarcitas y cuentas discoidales de Aragonita. En la cuadrícula NO del sector de excavación (C36/3), se detectó y excavó una lente de cenizas de $0,07 \mathrm{~m}$ de potencia que buzaba levemente hacia el sector SO del área excavada, a los $0,40-0,50 \mathrm{~m}$ de profundidad. Una vez dibujada y relevada se levantó completamente en bloques compactos y fue procesada utilizando el método de flotación. Se recuperaron contenidas entre las cenizas, restos de microfauna quemada y calcinada, semillas, cáscara de huevo e incluso una estructura floral en estado seco (Alavar 2017). Se realizó un fechado radiocarbónico por AMS sobre los restos de semillas carbonizadas del nivel III, el

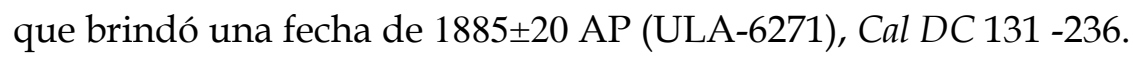

El tercer sector está localizado hacia el NO del segundo a una distancia de $16 \mathrm{~m}$ (UP B3, ver figura 4), y abarcó un total de 8 cuadrículas cubriendo una superficie de $8 \mathrm{~m}^{2}$. Aquí la cantidad de hallazgos es notoriamente menor que en la UP B2, estando ausentes muchos 
de los materiales que se encontraron en el sector 2. El material cerámico asciende a 399 fragmentos y los restos arqueofaunísticos son escasos $(\mathrm{N}=41)$. Se excavó en este lugar un entierro primario en fosa de un adulto masculino el que fue inhumado por debajo del piso fértil.

Sector UP C

Localizado hacia el extremo sur del sitio, sobre la terraza del río (Figura 4). Se abrieron 13 cuadrículas cubriendo una extensión de $13 \mathrm{~m}^{2}$. Se trata de otra área exclusiva de inhumación, habiéndose recuperado un total de 3 individuos; un sub-adulto y dos adultos femeninos. Todos ellos enterrados a diferentes profundidades. El entierro más profundo se encontró a 2,40 m. Los perfiles exhiben una gran homogeneidad y no se observan discontinuidades pedológicas o capas naturales de depositación. Esto se debe, probablemente, a la gran cantidad de carbonatos precipitados en una matriz netamente arenosa consolidada, donde las sales actuaron como agente cementicio (J. Kulemeyer com. pers.). La densidad de materiales arqueológicos es muy baja siendo mayor en los niveles superiores. El material cerámico asciende a 162 fragmentos pequeños de diferentes vasijas en el volumen total de sedimento removido $\left(32,2 \mathrm{~m}^{3}\right)$. Los restos arqueofaunísticos, son escasos $(\mathrm{N}=96)$ y poco diversos en comparación con el sector de excavación UP B2. Un fechado realizado al esqueleto del nivel III localizado a 2,07 m de profundidad, arrojó un

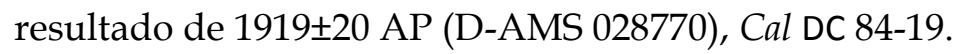

\section{Resultados}

Grilla de Sondeos: como se puede apreciar en la Figura 5, las mayores concentraciones de restos de malacofauna, ocurren al centro del área del sitio, particularmente las especies Pomacea y Bulimulus, y en coincidencia con otras concentraciones de artefactos (Figura 3). Resulta interesante notar que, las otras dos especies muestran un patrón de distribución más aleatorio, y hacia los límites de la grilla, las cantidades decrecen notablemente con las menores densidades afuera de los límites estimados del sitio arqueológico. En lo que respecta a las especies que pudieron ser identificadas, el mayor predominio corresponde a Pomacea aff. canaliculata ( $\mathrm{N}=247)$, seguido de las especies Bulimulus aff. apodemetes $(\mathrm{N}=51)$; Epiphragmophora aff. trigrammephora $(\mathrm{N}=33)$ y en último lugar Megalobulimus oblongus aff. lorentzianus $(\mathrm{N}=6)$ (Figura 6). Los niveles de profundidad de ocurrencia varían a lo largo y ancho del sitio, correspondiendo en algunos casos a los 30-40 cm y en otros muy superficialmente, $10 \mathrm{~cm}$. En el caso de las mayores concentraciones ocurren por debajo de los $30 \mathrm{~cm}$ de profundidad tanto en el sitio total como en las diferentes Unidades de procedencia excavadas (Figura 7 y Tabla 1 ). 

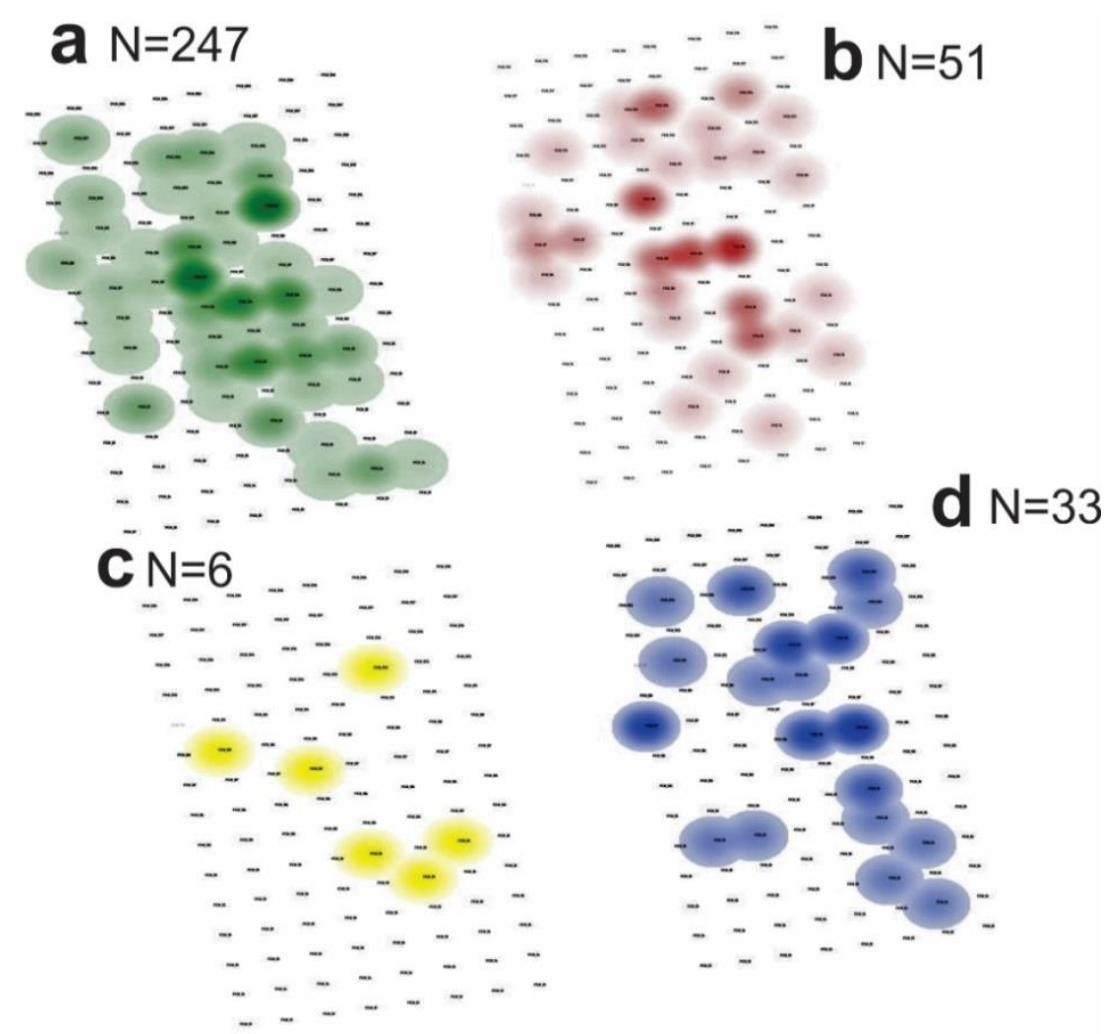

Figura 5. Mapa de calor que ilustra los sectores en donde ocurre la mayor abundancia de moluscos por especie en el sitio arqueológico. El color más intenso representa la mayor frecuencia. a) Pomacea; b) Bulimulus; c) Megalobulimus y d) Epiphragmophora.

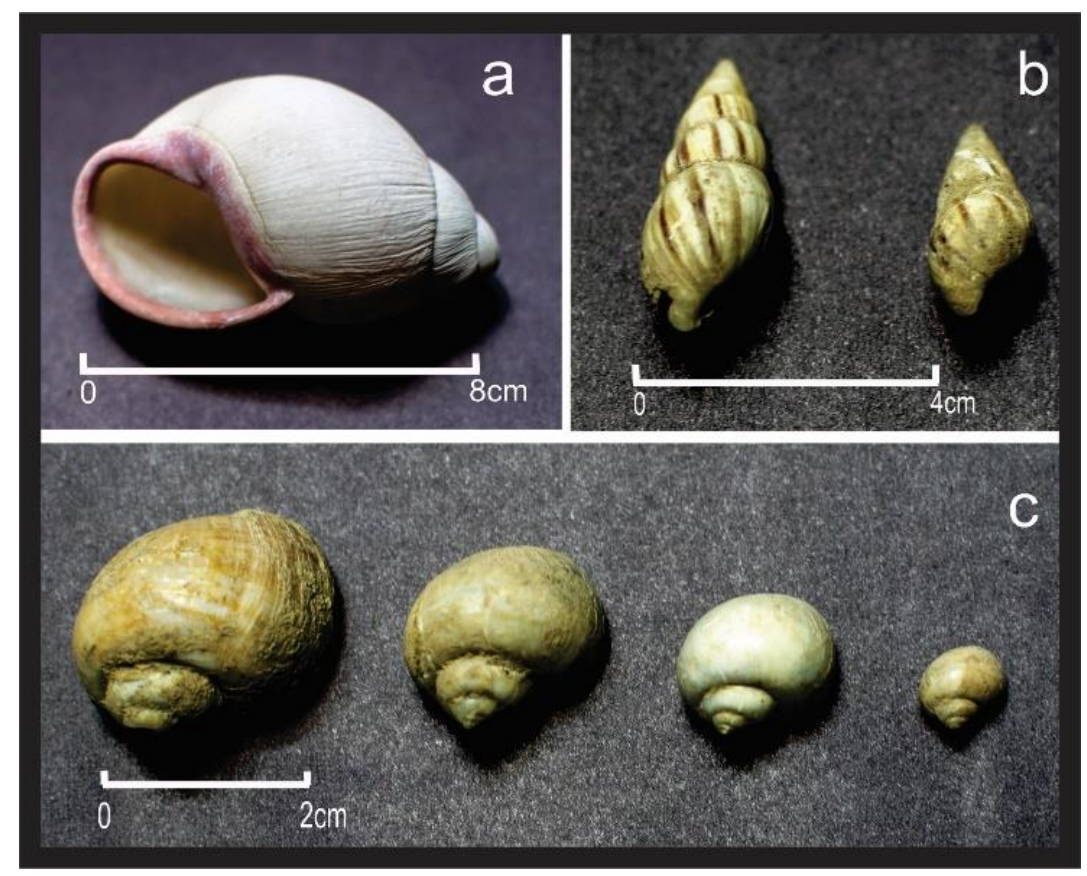

Figura 6. Especies recuperadas en el sitio. a) Megalobulimus oblongus aff. lorentzianus; b) Bulimulus aff. apodemetes; c) Pomacea aff. Canaliculata. 

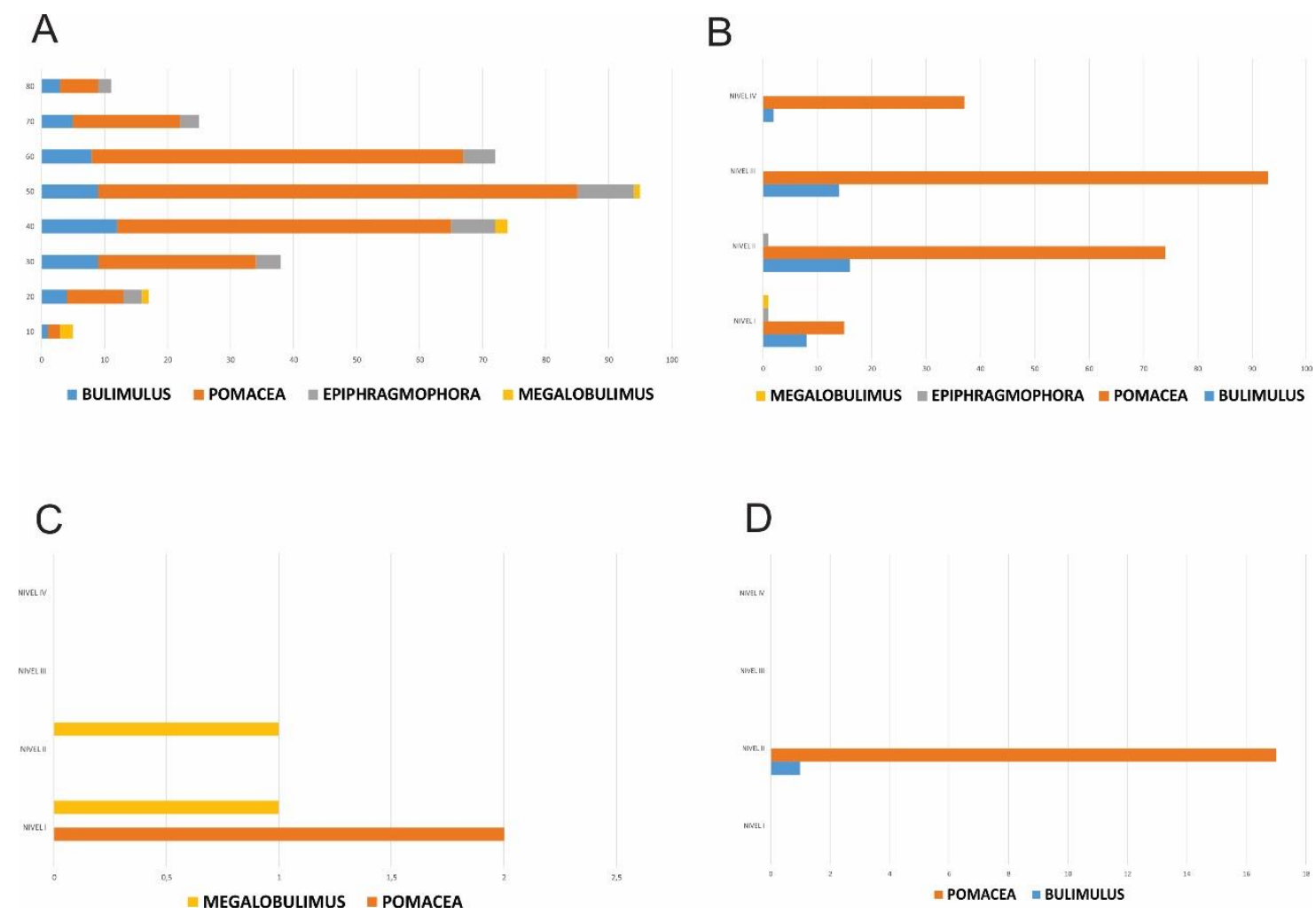

Figura 7. Abundancia de especies de moluscos de acuerdo a los diferentes niveles muestreados y excavados. A. Pozos de transectas. De abajo hacia arriba nivel 1 al 8. B. UPB 2; C. UPB 3; D. UPC.

UP B

Sector B1: la cantidad de moluscos en este lugar asciende a 11, en un total de volumen excavado de 5,2m³ (5.200 litros de sedimento extraído) (Tabla 1). Se trata de las especies Pomacea aff. canaliculata ( $\mathrm{N}=5)$; Megalobulimus oblongus aff. lorentzianus $(\mathrm{N}=4)$ y Bulimulus aff. apodemetes $(\mathrm{N}=2)$. En relación a otras faunas, predominan en los dos niveles superiores numerosas placas de edentados $(\mathrm{N}=685)$, algunas de ellas termoalteradas, vinculadas a un evento de quema evidenciado por una leve lente de carbón que discurre a lo largo del perímetro de los perfiles de la excavación con una potencia ínfima, menos de $1 \mathrm{~cm}$, sólo en las cuadrículas $2 / 1 ; 3 / 2$ y 4/3. Sin embargo, aún se desconoce si esta gran cantidad de placas obedece a algún fenómeno vinculado a procesos de formación de sitio o a un evento de depositación reciente, especialmente por tratarse del primer nivel de excavación y de un lugar donde acampan pescadores locales. Aún no se ha discriminado a cuantas especies de edentados corresponden los osteodermos recuperados (trabajo en curso). Sobre todo, considerando que en el lugar hay una asidua presencia de cazadores y pescadores actuales que acuden al río y eventualmente cocinan en el lugar. Estos animales son muy apreciados hasta la actualidad y suelen ser presa de cazadores que los consumen donde acampan. El resto total de otras faunas asciende a 205, con un NID de 58 (26,85\%) de la muestra total. 


\begin{tabular}{|c|c|c|c|c|c|c|c|c|c|c|c|c|c|c|}
\hline \multicolumn{15}{|c|}{ TRANSECTAS } \\
\hline \multicolumn{8}{|c|}{ NMI POR ESPECIE } & \multicolumn{7}{|c|}{ NISP POR ESPECIE } \\
\hline & $\mathbf{P}$ & $\mathbf{M}$ & $\mathbf{E}$ & B & \multicolumn{3}{|c|}{$\begin{array}{c}\text { TOTALES } \\
\text { PARCIALES } \\
\end{array}$} & $\mathbf{P}$ & $\mathbf{M}$ & $\mathbf{E}$ & \multicolumn{2}{|c|}{ B } & N/D & $\begin{array}{c}\text { TOTALES } \\
\text { PARCIALES } \\
\end{array}$ \\
\hline Nivel I & 2 & 2 & 0 & 1 & \multicolumn{3}{|c|}{5} & 0 & 0 & 0 & \multicolumn{2}{|c|}{2} & 6 & 8 \\
\hline Nivel II & 9 & 1 & 3 & 4 & \multicolumn{3}{|c|}{17} & 18 & 6 & 3 & \multicolumn{2}{|c|}{7} & 0 & 34 \\
\hline Nivel III & 25 & 0 & 4 & 9 & \multicolumn{3}{|c|}{38} & 55 & 6 & 4 & \multicolumn{2}{|c|}{16} & 0 & 81 \\
\hline Nivel IV & 53 & 2 & 7 & 12 & \multicolumn{3}{|c|}{74} & 100 & 11 & 8 & \multicolumn{2}{|c|}{34} & 2 & 155 \\
\hline Nivel V & 76 & 1 & 9 & 9 & \multicolumn{3}{|c|}{95} & 130 & 24 & 10 & \multicolumn{2}{|c|}{31} & 8 & 203 \\
\hline Nivel VI & 59 & 0 & 5 & 8 & \multicolumn{3}{|c|}{72} & 81 & 23 & 9 & \multicolumn{2}{|c|}{24} & 1 & 138 \\
\hline Nivel VII & 17 & 0 & 3 & 5 & \multicolumn{3}{|c|}{25} & 27 & 8 & 4 & \multicolumn{2}{|c|}{9} & 0 & 48 \\
\hline Nivel VIII & 6 & 0 & 2 & 3 & \multicolumn{3}{|c|}{11} & 12 & 3 & 3 & \multicolumn{2}{|c|}{4} & 1 & 23 \\
\hline TOTAL & 247 & 6 & 33 & 51 & & & & 423 & 81 & 41 & \multicolumn{2}{|c|}{127} & 18 & \\
\hline SECTOR/UP & $\begin{array}{c}\text { SUP. } \\
\text { EXCAVADA } \\
\end{array}$ & $\begin{array}{l}\text { VOL. } \\
\left(\mathrm{Mts}^{3}\right)\end{array}$ & $\mathbf{Z}$ & \multicolumn{5}{|c|}{ NMI POR ESPECIE } & \multicolumn{6}{|c|}{ NISP POR ESPECIE } \\
\hline & & & & $\mathbf{P}$ & $\mathbf{M}$ & $\mathbf{E}$ & B & \begin{tabular}{|c|} 
TOTALES \\
PARCIALES \\
\end{tabular} & $\mathbf{P}$ & $\mathbf{M}$ & $\mathbf{E}$ & B & N/D & $\begin{array}{c}\text { TOTALES } \\
\text { PARCIALES } \\
\end{array}$ \\
\hline $\mathrm{B} 1$ & $6 \mathrm{~m}^{2}$ & 5,4 & $0,90 \mathrm{~m}$ & 5 & 4 & 0 & 2 & 11 & 8 & 4 & 0 & 2 & 0 & 14 \\
\hline $\mathrm{B} 2$ & $16 \mathrm{~m}^{2}$ & 11,2 & $0,70 \mathrm{~m}$ & 495 & 2 & 2 & 42 & 541 & 722 & 90 & 2 & 72 & 11 & 897 \\
\hline B3 & $8 \mathrm{~m}^{2}$ & 5,6 & $0,70 \mathrm{~m}$ & 2 & 4 & 0 & 2 & 8 & 2 & 26 & 0 & 2 & 0 & 30 \\
\hline $\mathrm{C}$ & $13 \mathrm{~m}^{2}$ & 31,2 & $2,40 \mathrm{~m}$ & 32 & 1 & 2 & 1 & 36 & 60 & 9 & 2 & 2 & 0 & 73 \\
\hline TOTAL & & & & 534 & 11 & 4 & 47 & & 792 & 129 & 4 & 78 & 11 & \\
\hline
\end{tabular}

Tabla 1. Especies de moluscos recuperados en los distintos sectores de excavación y pozos de muestreo del sitio. Se indican las diferentes frecuencias y los valores de NISP y NMI. Referencias: P: Pomacea; M: Megalobulimus; E: Epiphragmophora; B: Bulimulus. 
Sector B2: sobre la base del NMI de moluscos, la cantidad total asciende a 541, discriminados por las siguientes especies; Pomacea aff. canaliculata (N=495); Megalobulimus oblongus aff. lorentzianus ( $\mathrm{N}=2)$; Bulimulus aff. apodemetes $(\mathrm{N}=42)$ y solo dos ejemplares de Epiphragmophora aff. trigrammephora, en un total de sedimento removido en $11,2 \mathrm{~m}^{3}(11.200$ litros) (tabla 1). En este sector algunos se presentan termoalterados $(\mathrm{N}=10)$, particularmente aquellos de la especie Pomacea, distribuidos principalmente alrededor de la lente de ceniza (Figura 8). Esta distribución es coincidente con la de otras arqueofaunas que nuevamente tienden a agruparse en los alrededores de ese mismo espacio. La importante abundancia además de otras arqueofaunas $(\mathrm{N}=2.745)$ entre las que se cuentan peces, edentados, aves, mamíferos y reptiles, algunos con marcas de corte, pulido y termoalteración, refuerza la idea de un área vinculada a la preparación y posiblemente consumo de alimentos. Al evaluar el tamaño de los moluscos, se observa que las dos especies más abundantes (Pomacea y Bulimulus) muestran rangos diferentes; mientras Pomacea presenta la mayor abundancia en el rango entre los 0,5 a 1,9 cm de largo por 1 a $2,5 \mathrm{~cm}$ de alto, Bulimulus muestra una mayor diversidad de tamaños (Figura 9).

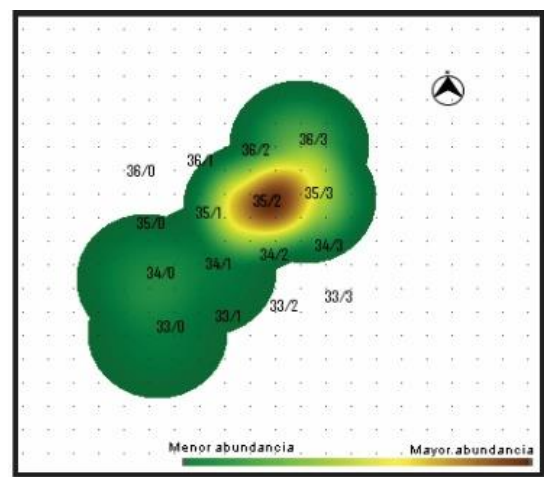

NIVEL I

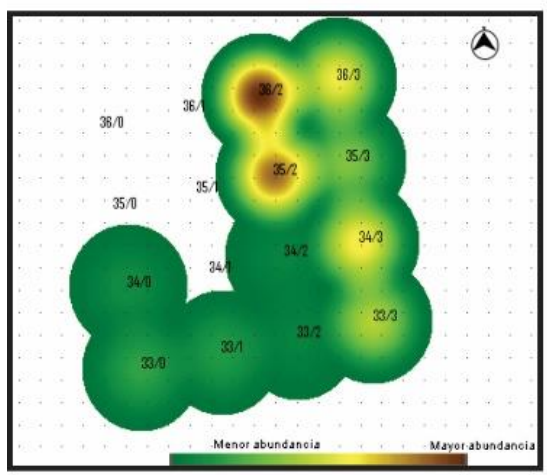

NIVEL II

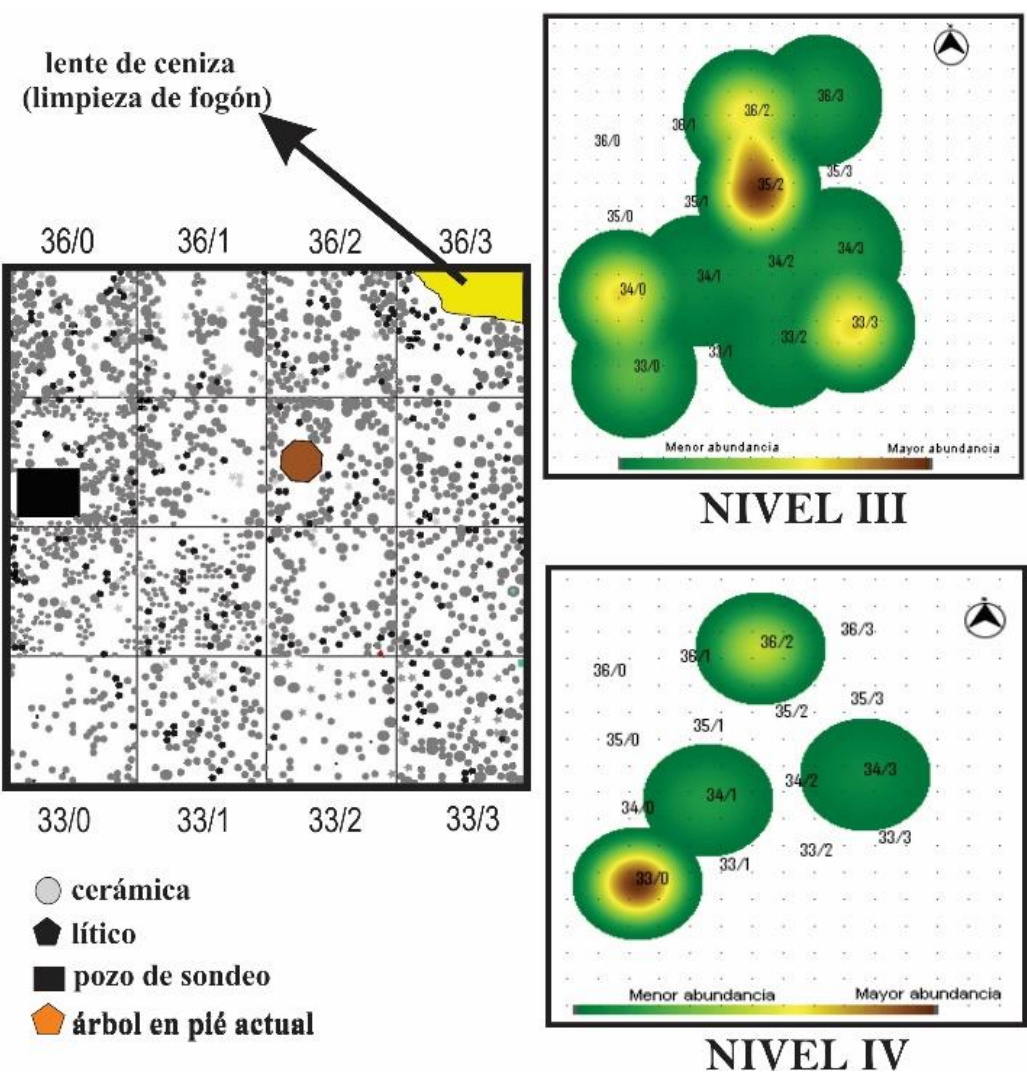

NIVEL IV

Figura 8. Planta de excavación de la UPB2. En los diferentes gráficos de mapas de calor se observan las concentraciones y distribución de los moluscos termoalterados desde el nivel I al IV. 

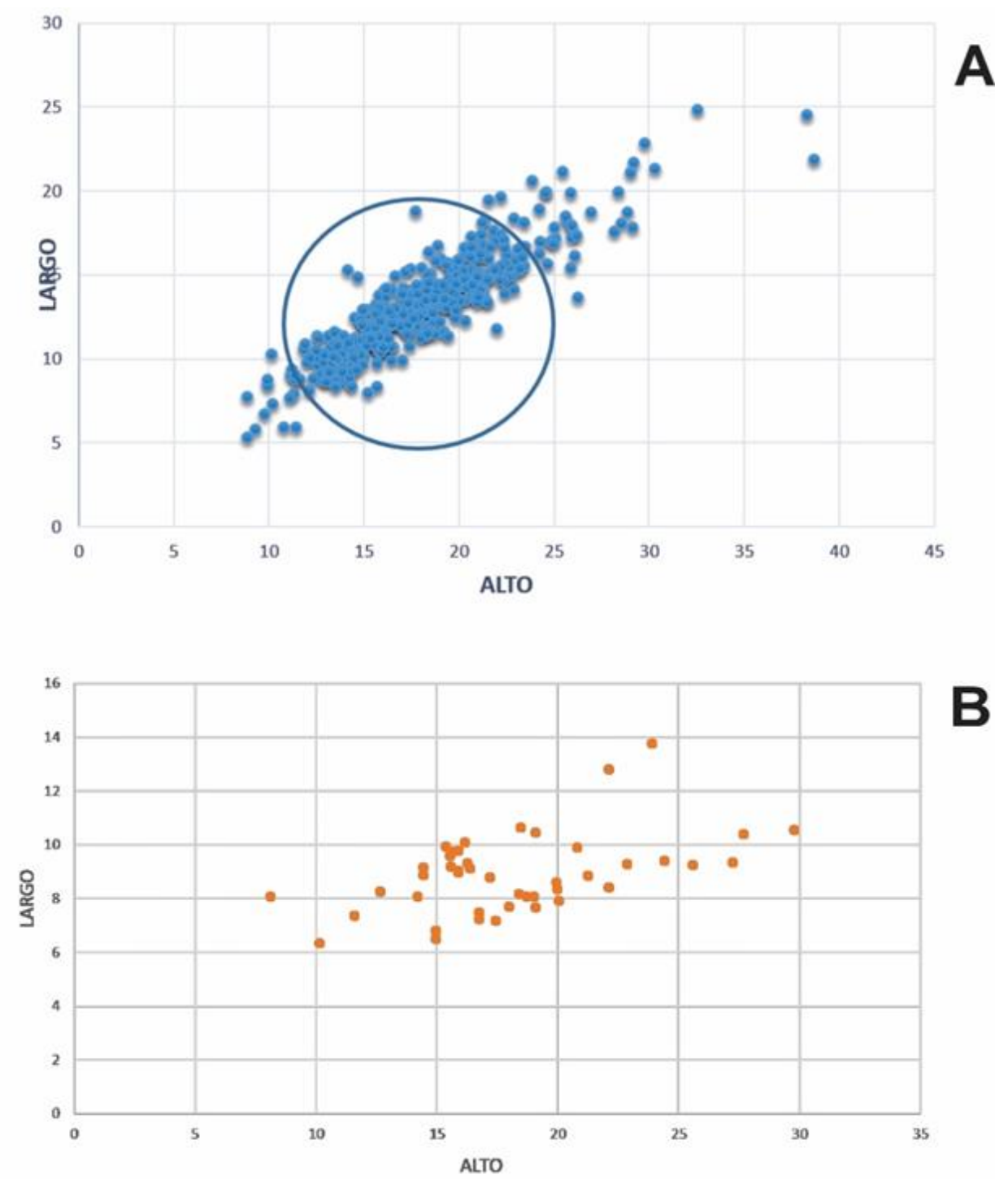

Figura 9. Gráfico de dispersión que compara pares de valores de medidas biométricas de las dos especies más abundantes en la UPB2. A. Pomacea y B. Bulimulus.

Sector B3: corresponde a un total de $8 \mathrm{~m}^{2}$ excavados y una cantidad de 8.000 litros de sedimentos extraídos. La frecuencia de los moluscos aquí es casi nula, con la recuperación de solo 8 ejemplares, 4 correspondientes a la especie Megalobulimus oblongus aff. lorentzianus; 2 de la especie Bulimulus aff. apodemetes y 2 Pomacea aff. canaliculata (Tabla 1), igualmente, la cantidad de los otros grupos faunísticos es escasa ( $\mathrm{N}=41)$. La distribución de la malacofauna en los diferentes niveles excavados solo se encuentra en dos cuadrículas, y tres de los ejemplares en una sola de ellas (C 40/22). Esta distribución parece a primera vista aleatoria, pero es interesante destacar que los Megalobulimus son los más frecuentes en este sector y a escasa distancia se produjo la inhumación de un individuo adulto. Hemos destacado en otra oportunidad, que el hallazgo de estos 
caracoles suele ser frecuente en contextos de inhumación (Ortiz y Vargas Rodríguez 2015). En esta oportunidad, se observa que su mayor número corresponde a dos de las tres áreas donde se han recuperado entierros en el sitio.

Sector C: ubicado sobre la terraza del río, se excavaron un total de $13 \mathrm{~m}^{2}$ con un volumen total de 13.000 litros de sedimentos extraídos. Los restos malacológicos de este sector corresponden a 32 Pomacea aff. canaliculata; 1 Megalobulimus; dos ejemplares de Epiphragmophora aff. trigrammephora y uno de Bulimulus aff. apodemetes (Tabla 1). En cuanto a su distribución en el total de los diferentes niveles excavados y considerando la importante profundidad de uno de los entierros $(2,45 \mathrm{~m})$, la mayor abundancia ocurre justamente a esa profundidad (nivel IV), y se distribuyen siguiendo el perfil de la barranca actual del río. Es notorio que solo se haya registrado un solo ejemplar de Megalobulimus considerando que se trata de otro sector exclusivo de inhumación.

No se han registrado hasta el momento en ninguno de los sectores excavados aparte de la UPA, ejemplares de Anodontites aff. trapesialis

\section{Discusión}

Debemos destacar la importante diferencia en lo que respecta a la abundancia relativa de restos de malacofauna tanto a escala del sitio, como de los diferentes sectores excavados. Sin embargo, en ninguno de los casos presentados, su distribución, concentración y frecuencia, se corresponde con bancos naturales.

Al considerar la tasa de depositación de los moluscos, es necesario tomar en cuenta los fechados obtenidos hasta el momento, cuyos resultados indican un período de 5 siglos en aproximadamente dos metros de potencia sedimentaria, que abarca desde comienzos de la Era Cristiana hasta el 500 DC (Ortiz et al. 2015). Al evaluar los datos obtenidos del muestreo de los pozos de sondeo en toda el área estimada de ocupación del sitio, podemos observar que la ocurrencia es baja, es decir, la tasa de depositación corresponde a una escasa proliferación de la malacofauna, sobre la base de la densidad en términos absolutos considerando la amplitud cronológica y la extensión del área ocupada. Sin embargo, una primera evaluación del total de las especies registradas, nos indica una elevada prevalencia de la familia Ampullariidae (aff. canaliculata). La abundancia relativa de este taxón en el sitio, considerando tanto los ejemplares de los pozos de muestreo como los recuperados de las áreas excavadas, es de $74 \%$, seguida por las otras especies con valores muy bajos (15\% Bulimulus; 9\% Epigramophora y 1\% para Megalobulimus). Los caracoles Pomacea son acuáticos, desarrollan su ciclo reproductivo en fuentes de aguas tranquilas (lagunas, encharcamientos, diques y arroyos) y suelen estar más activos durante la estación de verano (Vasquez Silva et al. 2011). En el invierno cierran la conchilla con el opérculo lo que les permite invernar. El tamaño de un ejemplar adulto oscila entre los 4,5 a $8 \mathrm{~cm}$. Son en general omnívoros, 
pero se alimentan preferentemente de una gran variedad de especies vegetales. También pertenecen a la clase de animales considerados comensales (Linares, 1976), es decir, son oportunistas, motivo por el cual han sido clasificados como plagas en aquellos países donde fueron introducidos por el daño a los cultivos y la incidencia sobre especies nativas (Rumi y Nuñez 2013). La mayor abundancia en los pozos de muestreo ocurre a partir de los $30 \mathrm{~cm}$, hasta los $60 \mathrm{~cm}$ donde su frecuencia comienza a disminuir (Tabla 1). Estas unidades estratigráficas de agregación coinciden con aquellas registradas en las diferentes Unidades de procedencia e interpretadas como pisos de ocupación. Los fechados obtenidos en el sitio, aunque abarcan un rango de más de 4 siglos, son más numerosos alrededor de los tres primeros siglos de la Era. Aquellos obtenidos en los niveles con mayor agregación de moluscos, corresponden a los dos primeros siglos DC.

En la UP B1, su bajísima frecuencia y distribución aleatoria, no indica que pudieran haber sido depositados por alguna clase de actividad humana intencional. Aquí, podría tratarse de animales intrusivos o que ingresaron al registro arqueológico, al momento de excavarse las fosas de inhumación y su posterior relleno, es decir, corresponderían al grupo tafonómico "intrusivo". Una explicación similar puede darse para la UP C, con una bajísima tasa de hallazgo a pesar de ser el sector con mayor cantidad de litros de sedimentos removidos (Tabla 1 ).

Otra situación muy diferente se registra en la UP B2, ya que se ha recuperado la mayor cantidad de ejemplares de moluscos con una dominancia notable de $P$. aff. canaliculata (Tabla 1). Sobre la base de su distribución espacial (alrededor de la lente de cenizas) y su creciente incidencia a mayor profundidad en los niveles excavados, estando su mayor frecuencia en el nivel III interpretado como el piso de ocupación, se infiere que su presencia se encuentra claramente vinculada con una acción antrópica. Muestran a su vez, un patrón de distribución espacial similar a los macrorrestos botánicos quemados y a otras arqueofaunas, que tienden a concentrarse en la proximidad y rodeando, la lente de ceniza localizada en la esquina NO de la cuadrícula $36 / 0$. Los carporrestos termoalterados tienen una frecuencia mayor en las cuadriculas $34 / 3,35 / 2,35 / 3,36 / 0$ y 36/2, y prácticamente se encuentran ausentes en las cuadrículas del extremo SE y NE de la excavación. Los moluscos termoalterados, aunque se agrupan alrededor de la lente de cenizas, implican solamente el 2,02\% de la muestra total. Es decir, que deben haber resultado quemados de manera accidental como parte del descarte en el fogón o al ser cubiertos con brasas y cenizas de su limpieza. El elevado número de ejemplares completos o parcialmente completos de Pomacea $(\mathrm{N}=495)$ en un sector acotado de $16 \mathrm{~m}^{2}$, no puede ser explicado como el resultado de eventos de acumulación natural. Por la propia etología de esta clase de animales que no suelen enterrarse a profundidad y además hibernar asociados a cursos de agua lénticos, sumado a la ausencia de señales que sugieran su transporte por 
factores naturales y su asociación con los pisos arqueológicos, se debe asumir un transporte y acumulación de origen antrópico. Sobre todo, si se compara con las frecuencias y distribuciones a nivel del sitio total. Sin embargo, el tamaño promedio indica que se trata de ejemplares juveniles que no han alcanzado aún su desarrollo completo. Esto indica que, aunque agentes naturales no sean los responsables del transporte, no es posible asegurar que su presencia en los contextos arqueológicos se deba a que fueron colectados por su utilidad como recurso comestible. Es en las colonias naturales donde existe la prevalencia de individuos jóvenes (Martin 1984), y en términos generales, solo los individuos adultos son seleccionados por los humanos para ser consumidos. Por lo tanto, su presencia debe ser explicada como resultado de algún otro factor que da cuenta de la abundante cantidad de esta clase de fauna asociada a los pisos arqueológicos. Una posibilidad es que, por tratarse de hospedadores oportunistas en áreas disturbadas antrópicamente, hayan incrementado su actividad en cercanías de las viviendas o lugares de producción hortícola. El desmonte de tierras antropogénicas, ya sea para campos agrícolas o para la construcción de edificios y montículos, crea nuevos entornos de borde mediante la modificación de paisajes y hábitats naturales (Clinton y Peres 2011). Como ya señalamos, algunas especies de moluscos, responden bien a la adaptación de entornos modificados. Otra alternativa posible, y no excluyente de la anterior, es que fueran involuntariamente ingresados al sitio y a las áreas de actividades domésticas, al estar parasitando algún recurso vegetal que era utilizado por los humanos. Eventualmente, podrían haber sido consumidos por estar disponibles, pero no como un recurso que fue colectado intencionalmente para ese uso, sino como algo probablemente excepcional y oportunístico. Es decir, que esta especie podría corresponder en el caso del sector UPB 2 a la combinación de dos grupos tafonómicos; el de "restos alimentarios" y "restos intrusivos penecontemporáneos".

El uso de caracoles dulceacuícolas con fines alimenticios en otros sitios arqueológicos en ambientes de tipo chaqueño, ha sido ampliamente demostrado (Santini y Lamenza 2013). Sin embargo, las medidas biométricas corresponden a una selección de ejemplares adultos y, además, la mayor acumulación se encuentra formando lentes bien delimitadas, situación muy diferente a la registrada por nosotros para el sitio analizado.

En el caso de la especie Bulimulus, que resulta ser la segunda en abundancia tanto a escala espacial del sitio arqueológico como en el sector de excavación UPB2; su amplia variación en las medidas biométricas indicarían diferentes estadios de vida, y la ausencia de evidencia de manipulación antrópica, sugiere que se trata de una especie que por sus hábitos oportunistas resultó en una mayor acumulación vinculada con los sectores de desechos, y posiblemente almacenamiento, de recursos vegetales asociados a un área de tareas domésticas. Es decir, corresponderían al grupo tafonómico "intrusivos penecontempoáneos". La misma clase de distribución, frecuencia y medidas biométricas, 
fueron registradas en el sector A del sitio, reforzando la primera interpretación sobre la presencia de esta especie en lugares vinculados con la preparación y/o consumo de alimentos (Ortiz y Vargas Rodríguez 2015).

La presencia de la especie Megalobulimus, indica otra situación. Aunque su frecuencia es muy baja en casi todo el sitio, si comparamos los valores del NMI (N=2) y del NISP $(\mathrm{N}=90)$ en el sector UPB2, observamos una elevada cantidad de fragmentos. Se trata de trozos de concha de pequeñas dimensiones que pudieron ser asignados a esta especie por la particular ornamentación de la valva y espesor. Considerando que se trata de una especie que presenta una concha robusta y resistente, los fragmentos podrían haber sido ingresados a los pisos fértiles arqueológicos por la acción del hombre. En este caso corresponderían al grupo tafonómico "de funcionalidad antrópica desconocida", ya que se trata de fragmentos que no presentan modificación o trabajo. Sin embargo, su frecuencia relativa es mayor que la de los Bulimulus, a pesar de que estos últimos presentan una concha frágil y quebradiza, por lo cual se esperaría una mayor tasa de fragmentación. Su mayor abundancia no puede ser explicada, por consiguiente, como resultado del pisoteo en un sector de intenso tránsito o actividad. Hay que destacar, que esta misma tendencia se mantiene a escala espacial del sitio arqueológico, y nuevamente asociada con los niveles estratigráficos entre los 30 a 60 cm de profundidad, es decir, en aquellos niveles de agregación penecontemporáneos al momento de ocupación del sitio. Una posibilidad es que los fragmentos hayan sido utilizados para la confección de cuentas discoidales. En el sector B2 se recuperaron 30 cuentas de Aragonita y 10 en el Sector A. Sin embargo, el origen de la materia prima aún no ha sido determinado, podría tratarse de material proveniente de fuentes termales o en su defecto, de la concha de los moluscos Megalobulimus (Boretto et al. 2018).

Finalmente, y evaluando las diferentes especies registradas, es posible ahondar en las condiciones paleoambientales de la época. La asociación entre especies de moluscos acuáticos y terrestres en conjunto con las medidas de abundancia relativa de individuos por especie y la distribución de sus frecuencias, pueden aportar valiosa información acerca de pulsos de mayor o menor humedad (Steffan 2007). En general, se suele asociar condiciones de mayor temperatura con un aumento de la humedad relativa, pero esta situación no es necesariamente contingente. Mientras otros indicadores de cambio climático pueden tener menos precisión, la asociación de taxones malacológicos es mucho más indicativa de condiciones locales y pequeñas variaciones. En este sentido se convierten en un "proxy" muy útil para detectar variaciones microambientales (Steffan 2007). En esta investigación, la importante dominancia de moluscos dulceacuícolas asociada a una muy baja frecuencia de especies de ambientes terrestres, indicarían un pulso más húmedo hace 2.000 años que en la actualidad (Figura 10). 


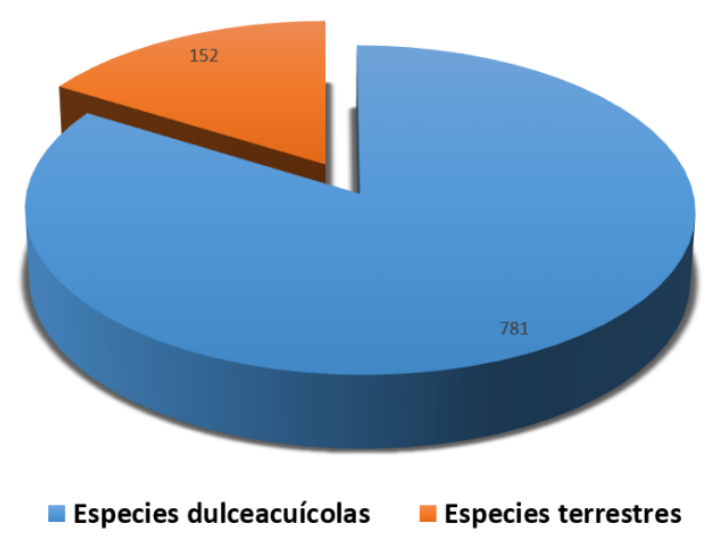

Figura 10. Cantidades totales de las diferentes especies en el sitio (acuáticas y terrestres).

Este dato coincide con los resultados obtenidos de los análisis antracológicos en el sitio (Ortiz et al. 2017), que indican en general, una situación de mayor humedad que la actual; es decir, un ambiente de chaco pedemontano ubicado hoy más al oeste.

Agradecimientos: A los editores por la invitación para participar en este volumen. A la Geóloga T. Chalabe por la diagramación de las figuras 2 y 3. A los administradores del Ingenio la Esperanza por su colaboración y predisposición para que los trabajos pudieran ser realizados en terrenos de su propiedad. Y finalmente, a los dos revisores externos por sus valiosos comentarios.

\section{Bibliografía citada}

Alavar, A.

2017. El registro arqueobotánico en el sitio Pozo de la Chola (2000-1500 a.p), valle de San Francisco, Jujuy. Usos de las plantas, ambiente y procesos tafonómicos. Tesis de Licenciatura. UNJu.

Bejega García, V.

2009. Arqueomalacología: metodología de análisis. Trabajo del $3^{\circ}$ ciclo, curso de Doctorado, Universidad de León. Pp112. www.academia.edu/1744383

Boretto, G.; Gordillo, S.; Izeta, A; Colombo, F.; Martinelli, M. y R. Cattáneo, 2018 Cuentas ornamentales en un contexto de cazadores-recolectores de la provincia de Córdoba: análisis mineralógico y microestructural de la concha de "Borus". Arqueología 24(1): 213-223. 
Clinton, J.M. y T.M. Peres

2011. Pests in the garden: testing the garden-hunting model at the rutherford-kizer site, Sumner county, Tennessee. Tennessee Archaeology 5(2): 131-141

Gautier, A.

1987. Tafonomic groups: how and why? Archaeozoología 1 (2): 47-52.

Gutiérrez Zugasti, F.I.

2008-2009. Análisis tafonómico en arqueomalacología: el ejemplo de los concheros de la región cantábrica. Krei 10: 53-74

Gordillo, S.

2010. Las almejas nacaríferas de la Cuenca parano-platense: patrimonio natural y cultural de Sudamérica. Cartilla de difusión $\mathrm{N}^{\circ}$ 15, Museo Provincial de Ciencias Naturales "Florentino Ameghino". Santa Fe, Argentina.

Linares, OF.

1976 "Garden Hunting" in the American Tropics. Human Ecology (4) 4: 331-349

Martín, S.

1984 Contribución al conocimiento de la biología de la familia Ampullariidae (Moll. Gasterópoda) en el río de La Plata. Tesis Doctoral, UNLP.

Miquel, S.E. y M.L. Aguirre.

2011. Taxonomía de los gasterópodos terrestres del Cuaternario de Argentina. Revista Española de Paleontología, 26 (2): 101-133.

Moreno Nuño, R.

1994. Análisis arqueomalacológicos en la Península Ibérica. Contribución metodológica y biocultural. Tesis Doctoral. Universidad Autónoma de Madrid.

Ortiz, G.

2007. La evolución del uso del espacio en las tierras bajas jujeñas (subárea del río San Francisco). Tesis de Doctorado. UNCo.

2015. Avances y nuevas perspectivas en la arqueología del pedemonte de Jujuy (valle de San Francisco), Argentina. En el Corazón de América del Sur III: Arqueología de las Tierras Bajas de Bolivia y zonas limítrofes (ed. por S. Alconini y C. Jaime Betancourt), pp. 195-212. Museo de Historia de la UAGRM, Santa Cruz, Bolivia. 
Ortiz, G. y L. Nieva

2014. Rituales y memorias del pasado. Practicas funerarias en la región del rio San Francisco, Jujuy, Argentina. Antropología Biológica y estudios del comportamiento mortuorio de los pueblos prehispánicos del Noroeste Argentino (ed. Por V. Seldes y M.S. Gheggi), pp. 149174. Fundación de Historia Natural Félix de Azara, Buenos Aires, Argentina.

Ortiz, G. y N. Vargas Rodríguez

2015. Más allá del artefacto. Aproximación al ambiente y estrategias de uso de moluscos en la región del Valle de San Francisco, Jujuy (0 al 500 dc). Arqueomalacología. Abordajes metodológicos y casos de estudio en el Cono Sur (ed. Por H. Hammond y M.A. Zubimendi), pp. 59-78. Fundación de Historia Natural Félix de Azara, Buenos Aires, Argentina.

Ortiz, G., C. Heit, L. Nieva, F. Zamora, N. Batallanos y F. Chapur 2015. Pensando al formativo desde la región pedemontana de las yungas de Jujuy. Crónicas materiales precolombinas. Arqueología de los primeros poblados del Noroeste Argentino (ed. por A. Korstanje, M. Lazzari, M. Basile, F. Bugliani, V. Lema, L. Pereyra Domingorena, y M. Quesada, M.), pp. 695-720. SAA, Buenos Aires, Argentina.

Ortiz, G. y V. Killian Galván

2016. El consumo como vía para comprender economías mixtas. Su aplicación al sur del valle de San Francisco, región pedemontana de Jujuy (Argentina). Las montañas tropicales y valles contiguos de los andes sud-centrales (ed. por S. Alconini), pp. 263-282. Plural, Bolivia.

Ortiz, G., R.S. Ramos, A. Alavar

2017. Fire, rituals and domesticity. Forest resource management in the subAndean region of Jujuy, Argentina (2000 BP): First anthracological evidence. Journal of Anthropological Archaeology, 47, 96-108. doi:10.1016/j.jaa.2017.04.002

Ortiz G., F. Paz, B. Zenteno, S. Zúñiga, L. Nieva

2018. Estudio de sub-adultos de la cuenca del río San Francisco, provincia de Jujuy, Argentina (0-500 dc). Revista Argentina de Antropología Biológica, Vol. 20, N²: 1-15. Universidad Nacional de La Plata. doi:10.17139/raab.2018.0020.02.01

Ramírez, R., V. Borda, P. Romero, J. Ramírez, C. Congrains, J. Chirinos, P. Ramírez, L.E. Velásquez y K. Mejía

2012. Biodiversidad y endemismo de los caracoles terrestres Megalobulimus y Systrophia en la Amazonia occidental. Rev. Perú. Biol. 19 (1):59-74. Facultad de Ciencias Biológicas UNMSM 
Rumi, A. y V. Nuñez

2013. Gasterópodos continentales de importancia sanitaria en el noroeste de Argentina. En Salomón, ODA. Y A. Rumi (eds.) Moluscos de interés sanitario en Argentina, pp. 7-39. INMET, Puerto Iguazú, Argentina.

Santini, M. y G. Lamenza

2013. Estudios de subsistencia en el Chaco húmedo argentino. En el corazón de América de sur 3. Arqueología de las tierras bajas de Bolivia y zonas limítrofes (ed. por S. Alconini y C. Jaime Betancourt), pp175-194. Biblioteca del Museo de Historia, Universidad Autónoma Gabriel Moreno, Santa Cruz de la Sierra, Bolivia.

Steffan, P.

2007. Análisis paleoambiental sobre la base del estudio malacológico del sitio Paso Otero 1 (región pampeana, Argentina). Intersecciones en Antropología 8: 173-184.

Vázquez Silva, G., T. Castro Barrera, J. Castro Mejía y G.D. Mendoza Martínez 2011. Los caracoles del género Pomacea (Perry, 1810) y su importancia ecológica y socioeconómica. Contactos 81:28-33. México

Villamarzo, E.

2010. Arqueomalacología del sitio La Esmeralda (Rocha, Uruguay) Comunicaciones de la Sociedad Malacológica del Uruguay 9 (93): 215-230. 